\title{
Specific Immunotherapy
}

\section{Spaskova $\mathbf{V}^{*}$}

Department of Dermatovenerology, P.I.Center for Traditional Chinese Medicine- Shtip, Republic of Macedonia

*Corresponding author: Spaskova V, Department of Dermatovenerology, P.I.Center for Traditional Chinese Medicine- Shtip, R.Macedonia, Republic of Macedonia, Email: vaskaspaskova@yahoo.com

Received date: May 15, 2018; Accepted date: May 21, 2018; Published date: May 28, 2018

Copyright: @ 2018 Spaskova V. This is an open-access article distributed under the terms of the Creative Commons Attribution License, which permits unrestricted use, distribution, and reproduction in any medium, provided the original author and source are credited.

Keywords: Allergens; Specific immunotherapy; Allergen-specific immunotherapy

\section{Introduction}

Common expressions of specific immunotherapy are: desensitization, hyposensitization, Immunotherapy. But according to the WHO recommendation, the most appropriate term seems to be "allergenic vaccination" [1].

In 1911, Noon published a short sentence for the treatment of pollen disease by applying a Phleum pretense aqueous extract. The cooked allergen was diluted and the solution obtained was tested by dropping into the patient's eye by following the conjunctival reaction. In the same year, Freeman published a letter about the success of immunotherapy in 20 people with pollenosis.

In 1918, Cooke reported that allergic diseases such as asthma and pollen arose as a result of the generation of antibodies after exposure to allergens [1].

What Is Specific Immunotherapy? Specific immunotherapy (SIT) is the basic method of treatment in allergology. Its most important significance is the discontinuation of the allergic process at the very beginning, and has much greater advantages over the drug treatment and approach to the disease, changes the direction of allergic diseases, significantly improves the quality of life of patients in different percentages (60-90\% depending on the type of sensitization), achieves permanent cure or long-term remission. Application schemes vary with different types of allergens and are individualized according to the patient's effect and tolerance [2].

\section{Common expressions of specific immunotherapy are:}

- desensitization

- hyposensitization

- Immunotherapy

but according to the WHO recommendation, the most appropriate term seems to be "allergenic vaccination".

\section{History of SIT}

SIT has been implemented in the treatment of allergic diseases for almost 100 years. In 1911, Noon published a short sentence for the treatment of pollen disease by applying a Phleum pretense aqueous extract. The cooked allergen was diluted and the solution obtained was tested by dropping into the patient's eye by following the conjunctival reaction. In the same year, Freeman published a letter about the success of immunotherapy in 20 people with pollenosis.
In 1918, Cooke reported that allergic diseases such as asthma and pollen arose as a result of the generation of antibodies after exposure to allergens. At the end of the $60 \mathrm{~s}$ of the last century, the development of immunological methods and the use of RAST and the determination of specific IgE, which are key in the appearance of allergic diseases, has been developed. In 1978, Hunt gave a report on the success of SIT in patients who had anaphylactic reactions after a bee or axillary bite that subsequently resulted in the introduction of SIT in routine therapy in the treatment of allergic to insect stings.

In 1994, "American Academies of Allergy and Immunology" adopted guidelines with recommendations for the application of SIT with the main goal of avoiding and minimizing unwanted occurrences from it.

\section{What is Specific Immunotherapy?}

Specific immunotherapy (SIT) is the basic method of treatment in allergology. Its most important significance is the discontinuation of the allergic process at the very beginning, and has much greater advantages over the drug treatment and approach to the disease, changes the direction of allergic diseases, significantly improves the quality of life of patients in different percentages (60-90\% depending on the type of sensitization), achieves permanent cure or long-term remission. Application schemes vary with different types of allergens and are individualized according to the patient's effect and tolerance.

SIT in practice is the application of gradually increasing doses with the relevant allergenic vaccines, reaching the dose that is effective in improving the symptoms associated with exposure to the incriminated allergens.

SIT is the only treatment that affects the natural course of allergic diseases, designed for patients who have demonstrated specific IgE antibodies to clinically relevant allergens.

\section{How to Make an Allergy?}

Enemies from the environment are constantly attacking our body by breaking the homeostasis. This disorder of immunological homeostasis can be manifested differently.

1. Hypersensitivity-overactive responses to allergens

\section{Immunodeficiency-insufficient}

\section{Inappropriate to your own proteins-autoimmune diseases}

Under normal conditions, the immune system successfully cures removal of the antigen and ends with a small inflammatory reaction and without damage to its own tissue.

In certain conditions, the inflammatory reaction may be excessive and manifests itself with significant tissue damage, the onset of illness, 
and even death. This damaging and inadequate immune response is called hypersensitivity.

\section{Mechanism of Action of Specific Immunotherapy}

SIT (hyposensitization, desensitization) has been used in the treatment of allergic diseases since 1911. It is a multiple application of the specifically responsible allergens of the patient with a gradual increase in the dose in order to reduce allergic symptoms. In this way, it is necessary to achieve immune tolerance and to reduce the severity of allergic reactions of type I or to completely remove it. With the application of SIT it is necessary to establish a balance between effector and regulatory $\mathrm{T}$ lymphocytes which results in the establishment of peripheral tolerance, and immunotherapy itself leads to a change in Th2/Th1 lymphocyte ratio in favor of Th1.

The basic mechanism of action of specific immunotherapy is the generation of peripheral tolerance of the allergen, that is, the remeasurement of the peripheral $\mathrm{T}$ lymphocytes and the sensitization of the allergen do not respond with an inflammatory allergic response.

In addition, the stimulation of the production of regulatory $\mathrm{T}$ lymphocytes, which with its suppressive action prevent cell proliferation and cytokine production at several levels of the development of the inflammatory cascade. The process of creating regulatory $\mathrm{T}$ lymphocytes throughout the SIT begins so that the growing allergen dose stimulates the creation of allergenic specific regulatory lymphocytes similar to $\mathrm{T} 1$ regulatory lymphocytes.

These cells produce large amounts of IL10 as the main effector cytokine through which they achieve their suppressive effect. Although they produce IL10 as well as T1 regulatory lymphocytes, most of these cells are phenotypic CD4CD25, which differ from CD4CD25 T1 regulatory lymphocytes. The created regulatory $\mathrm{T}$ lymphocytes are reprogramming further developments throughout the SIT acting suppressively to different cells involved in the allergic inflammatory response (antigen recognition cells, Th lymphocytes, B lymphocytes, effector cells of the wound and late stage of the allergic reaction as well as the cells of individual tissues where the allergic reaction occurs).

Starting from the level of the allergen recognition cells, regulatory $\mathrm{T}$ lymphocytes suppress dendritic cells responsible for the production of effector Th2 lymphocytes and encourage non-inflammatory dendritic cells that, by means of IL10, mediate the further formation of new regulatory TreP1-lymphocytes. With the maturation of dendritic cells in the presence of IL10, they arrive at incomplete differentiated shapes expressed as a reduced expression of the MHC II molecule as well as certain costimulatory and adhesion molecules and thus obtain tolerant properties.

These cells have the ability to act suppressively on Th1 and TH2 lymphocytes. For the occurrence of regulatory lymphocytes that are able to further suppress Th2 immune response in people with allergic disease, simultaneous presence of tolerogenic dendritic cells and TGNbeta is required. In fact, the regulatory T lymphocytes that occur in SIT are those that simultaneously produce IL10 and TGF-beta so they can provide a micro-balance for further targeting of the immune response to the creation of new regulatory rather than effector Th2 lymphocytes. regulatory instead of effector Th2 lymphocytes.

The mechanisms by which regulatory $\mathrm{T}$ lymphocytes during suppression of the immune response suppress the immune response are anergy as well as their increased apoptosis. Decreasing the IgE/IgG ratio over SIT is a consequence of the prediction of regulatory $\mathrm{T}$ lymphocytes. By IL10 mediation, these lymphocytes directly affect isotype transfer to B lymphocytes resulting in reduced formation of $\mathrm{IgE}$ antibodies. However, a significant reduction in the $\mathrm{IgE} / \mathrm{IgG}$ ratio occurs only after several months after the onset of CIT (although the regulatory cells occur within days).

This time difference can not be explained by the length of the antibody half-life, which for Ig is 2.5 days and for IgG for 23 days. It is thought that the existence of long-lived plasma cells in the bone marrow is responsible for this difference. The generated IgG4 antibodies have specific structural and functional features. Due to the low affinity for Fc-ipsilon receptors and the $\mathrm{Clq}$ component of the complement do not lead to cell activation and complement.

They are not able to create large immunocomplexes and functionally act as blocking antibodies. By binding to the allergen, it is prevented that the allergen binds to IgE on the surface of the mast cells and basophils, thereby preventing the subsequent degranulation. In a similar way, they also inhibit the binding of the B cell B cell allergen and the further generation of IgE antibodies. In both cases, the IgG4 antibody activity may be increased by binding the IgE-allergen-IgG4 complex to inhibitory receptors, thereby canceling simultaneous signaling by IgE receptors, or B cell receptor.

Furthermore, IgG4 antibodies also prevent the binding of an IgE allergen bound to high or low-alpha receptor cells responsible for antigen presenting. In this way they stop the process of facilitated presentation of allergen by IgE to TH2 lymphocytes. The effects of regulatory $\mathrm{T}$ lymphocytes encompass an increase in the sensitivity threshold of mast cells and basophils, a reduction in the subsequent release of inflammatory mediators from these cells, and a reduced activation of eosinophils.

Among the effects that are relevant to the late stage of the allergic reaction include reduced innervation of the nasal and bronchial mucosa with mast cells and eosinophils, and consequently reduced hyperreactivity of these mucous membranes to specific stimuli.

\section{Indications for the Use of ASIT}

Allergy is an important health problem in industrialized countries and its prevalence is constantly rising: In 1906, when Clemens von Pirquet introduced the term "allergy" to describe a myriad of pathological reactions to the body caused by contact with antigens (less than $1 \%$ of the population were affected). Today, more than $25 \%$ of residents of industrialized countries suffer from allergic rhinitis, conjunctivitis, asthma, dermatitis, or food allergies.

Allergic symptoms can be alleviated by using corticosteroids, antihistamines, or leukotriene antagonists, however, only immunotherapy is able to change the course of allergic diseases and lead to long-term improvement that has been prolonged for years after discontinuation of this treatment. The treatment of allergic (atopic) diseases is based on the following basic principles:

- Avoiding allergens

- pharmacotherapy

- Allegene specific immunotherapy

- Education of patients

ASIT is used in the treatment of allergic diseases due to type 1 hypersensitivity reaction and begins with a type 1 allergic reaction based on: reaction type 1 on the basis of:

- An assimilated collection 
- Physical finding

- Skin tests (inhalatory and nutritive allergens)

PRICK test is the right choice in the diagnosis and treatment of allergic diseases. A drop of commercially-standardized allergenic extract is applied to the volar side of the forearm and lance with the lancet so that a prick and lift cannot be shown, because in that case we are already in the derm.

In the epidermis there are no mast cells, but by scratching the horn layer of the epidermis, the allergenic extract penetrates the dermis. Positive skin reaction is due to the presence of specific IgE on the surface of mast cells, the amount of the allergen and the reactivity of the skin of histamine. Histamine contributes to vasodilatation (erythema) and local edema (urtica) in the dermis.

Allergens are applied to each other at a distance of at least $1.5 \mathrm{~cm}$ so that the distance of the latter is $3 \mathrm{~cm}$ from the jackpot. After the top of the $1 \mathrm{~mm}$ lance, the drop after 1 minute can be easily wiped and read after 15 minutes. Always have positive control with $1 \%$ histamine and negative with buffered saline. Regardless of the erythema in the interpretation of the test, only the urticaria is mundane.

The size of the urticate is determined as the average of the longest and shortest diameters $(\mathrm{d} 1+\mathrm{d} 2): 2$. A positive test is considered to be a wrinkle $>3 \mathrm{~mm}$ (for a standardized solution), if no standardized solution can be used natural allergen sources (apple, milk) so that the tip of the lancet immerses in the grocery and then makes a skin nail. The amount of the allergen entered is usually less than 1 microgram.

\section{Determine the Specific IgE}

The total IgE alone does not provoke atopia, but however, the high $\mathrm{IgE}$ indicates the tendency of a type of hypersensitivity to an allergen. The optimum time for blood tests to determine the specific IgE is after 2-3 weeks, up to 6 months after the onset or worsening clinical picture (especially important for hypersensitivity to drugs and stings.

\section{Allergen-Specific Immunotherapy is an Effective Treatment for}

- Hymenoptera toxin allergy (bees, axes, ants, stag)

- Allergic rhinocytic conjunctivitis, caused by: -Pollen (oak, olive, birch, clear, poplar, linden, grass)

Treatment is more successful when using a large number of different types of allergens (treatment with one allergen is abandoned)

- Domestic dust with dermatophagoids

- Animals

- Asthma

- Pollen

- Homemade dust with Dermatophagoides pteronissimus.

- Animals/pets (in special cases).

- An allergy to mold (not referring to symptoms associated with damage to habitats with water, which are usually irritating symptoms).

- Food allergy (cow's milk, eggs, peanuts)

- Professional allergies (latex)
The decision to continue allergen-specific immunotherapy should be done taking into account individual patient factors, such as the extent to which symptoms can be reduced, the amount and type of drugs needed to control the symptoms, the adverse effects of pharmacological treatment and patient preferences. Because this form of therapy carries the risk of anaphylactic reactions, it should be prescribed only by doctors who are adequately trained in the treatment of allergy.

Immunotherapy or hyposensitization is a specific treatment method in allergology. The method is based on a procedure used to obtain an increase in IgG antibodies, thereby preventing mast cell degranulation.

This method does not require the use of systemic corticosteroids, antihistamines, and bronchodilators for a long period of time for patients.

\section{Types of ASIT}

1. Sublingual

2. Subcutaneous

3. Nasal

4. Oral (in nutritive allergens)

5. Short-term immunotherapy (currently used in pollen allergies and allergens with a high degree of purification that are capable of providing rapid hyposensitization are used)

6. Immunotherapy with allergoids

7. Recombinant vaccines

8. Anti-IgE antibodies in combination with ASIT

\section{Discussion}

It is a multiple application of the specifically responsible allergens of the patient with a gradual increase in the dose in order to reduce allergic symptoms. In this way, it is necessary to achieve immune tolerance and to reduce the severity of allergic reactions of type I or to completely remove it. The basic mechanism of action of specific immunotherapy is the generation of peripheral tolerance of the allergen, that is, the re-measurement of the peripheral T lymphocytes and the sensitization of the allergen do not respond with an inflammatory allergic response. In addition, the stimulation of the production of regulatory $\mathrm{T}$ lymphocytes, which with its suppressive action prevent cell proliferation and cytokine production at several levels of the development of the inflammatory cascade [3].

Allergen-specific immunotherapy is an effective treatment for: hymenoptera toxin allergy (bees, axes, ants, stag), allergic rhinocytic conjunctivitis, caused by pollen (oak, olive, birch, clear, poplar, linden, grass). Treatment is more successful when using a large number of different types of allergens (treatment with one allergen is abandoned): domestic dust with dermatophagoids, animals, asthma, pollen, homemade dust with dermatophagoides pteronissimus, animals/pets (in special cases), an allergy to mold (not referring to symptoms associated with damage to habitats with water, which are usually irritating symptoms), food allergy (cow's milk, eggs, peanuts), professional allergies (latex) [4].

Immunotherapy or hyposensitization is a specific treatment method in allergology. The method is based on a procedure used to obtain an increase in IgG antibodies, thereby preventing mast cell degranulation [5]. 
Citation: Spaskova V (2018) Specific Immunotherapy. J Allergy Ther 9: 278. doi:DOI: 10.4172/2155-6121.1000278

Page 4 of 4

\section{References}

1. Lockey RF, Ledford DK (2014) Allergens and Allergen immunotherapy (5thedn). Taylor \& Francis CRC Press pp: 576.

2. William EP (2012) Fundamental Immunology (7thedn). Wolters Kluwer Health/Lippincott Williams \& Wilkins pp: 1312.
3. Kindt TJ, Goldsby RA, Osborne BA (2000) Kuby Immunology (4thedn).W. H. Freeman and Company.

4. Abbas A, Lichtman AH, Pillai S (2014) Cellular and Mollecular Immunology (8thedn) Nature Publishing Group.

5. Blaser K (2008) T Cell Regulation in Allergu, Asthma and Atopic Skin Diseases. Karger Medical and Scientific Publishers pp:225. 